\title{
A helping hand with language learning: teaching French vocabulary with gesture
}

\author{
Alison Porter \\ Department of Modern Languages, University of Southampton, Southampton, \\ UK. \\ Email: amp1g09@soton.ac.uk.
}

\begin{abstract}
Finding ways to make language teaching practices both active and effective is of great importance for young learners. However, extending the foreign language production of young learners in instructional settings beyond the naming of objects is often challenging. The memorisation abilities of very young learners (children aged 5-7) sometimes appear limited and attrition is a major issue, given the onceweekly teaching sessions which are a common model for UK primary modern foreign language instruction. This study explored the effectiveness of gestures, as a form of elaborated encoding for young learners, in aiding target language memorisation and slowing attrition through the implementation of a strict teaching protocol and a bespoke pedagogical tool. Findings show significant advantage for short-term retention of a story told with both gestures and pictures when compared with a story told with pictures only. Delayed post-test scores for the gestured story demonstrate a greater rate of attrition from a higher initial mean score than the non-gestured story. This study will therefore assert that gestures boost memorisation due to retrieval cues and richer memory traces. However, it will also note that, when considering longer-term retention, a higher rate of attrition for the gestured story shows that a richer trace alone is not enough. In other words, whilst elaborated processing enhances memorisation, even richer traces need refreshing through repetition and retrieval practice.
\end{abstract}

\section{Introduction}

Gesture, to aid memorisation of lexical items, is an often-practised strategy amongst foreign language teachers and has been both recommended in government advice and observed in recent research. In England, the Key Stage 2 Framework for Languages (DfES 2005: 19) refers repeatedly to the wider use of gesture/physical response in language teaching, stating, for example, that in year 3 (ages 7-8) children should be able to 'use physical response, mime and gesture to convey meaning and show understanding' (Oracy objective 3.4). The use of actions, rhymes and games to aid memorisation are also recommended as part of 
a Year 3 formative assessment strategy (Asset Languages and CILT 2010: 17). Recent research conducted in primary school classrooms in England (children aged 5-11) has noted that both teachers and children use and value physical gesture to aid memorisation and visualisation in second language learning (Cable, Driscoll, Mitchell and Holmes 2010: 81, 86, 94 and 99). However, there seems to be little empirical evidence exploring the nature of this strategy's success or identifying its possible limitations (Tellier 2008).

This study's fundamental premise is that gesture supports memorisation in language learning through enhanced or elaborated processing and therefore creates a more durable and more accessible memory trace for new language. In practical terms, such traces should result in higher recall (production) and lower attrition (loss) of lexical items learnt with gestures as supports. The theoretical framework for this paper will set out ideas relating to levels of processing in memorisation (Craik and Lockhart 1972), a model of working memory (Baddeley 2003) which posits the idea that visual and non-visual information can be processed simultaneously, and research relating to memorization of 'events involving actions' (Cohen, 1989: 60) which holds that enactment might enhance accessibility of traces (Cohen and Otterbein 1992: 118), is non-strategic (Cohen 1981, 1983) and also creates more distinctive and richer traces (Bäckman and Nilsson 1984, 1985). The multi-faceted role of gesture in language use will be acknowledged, and existing research relating to the idea that gestures can aid language learning will be examined.

\section{Background \\ Levels of processing}

The idea of levels of processing was encapsulated by Craik and Lockhart (1972) who posited that remembering was more about the residual 'after-effects of processing' than hypothetical short- and long-term memory structures (Baddeley 1997: 115). In this view, differing traces 'persist for different lengths of time': initial traces are 'superficial and shallow' (for example, physical or sensory features such as lines, pitch, brightness), but subsequent deeper processing involving perhaps pattern recognition and extraction of meaning can produce 'depth of processing' which, in turn, leads to 'more elaborate, longer lasting and stronger traces' (Craik and Lockhart 1972: 675). Baddeley concludes that structure in memory (short- and long-term storage) is better defined by the level of processing involved than by ideas relating to unitary 'stores'. Short-term memory is characterised by 'maintenance rehearsal' which holds information but does not transform it, whilst long-term memory is concerned with 'elaborative rehearsal' which increases the depth of encoding (Baddeley 1997: 116). He also suggests that the key to learning is organisation and consolidation (or processing), in other words, 'what the subject does with the material to be remembered' (Baddeley 1997: 107) and that richer traces improve learning (Baddeley 1997: 120-121). Hulstjin too asserts that 'richness of encoding' is widely accepted as leading to higher retention of lexical items (Hulstjin 2001: 270) but adds that rich elaborate processing might not be enough and that new information must also be reactivated by rehearsal activity to produce a more lasting trace (Hulstjin 2001: 269). 


\section{Multi-component working memory}

Following Craik and Lockhart's examination of memory from an input or encoding perspective, Baddeley and Hitch proposed a working memory model with three separate components which might support such memorisation processes. The model comprised: the central executive, the visuo-spatial sketchpad and the phonological loop which could deal simultaneously with different kinds of input (Baddeley and Hitch 1974: 47-90). The visuo-spatial sketchpad could integrate spatial, visual and possibly kinaesthetic information into a unified and adaptable representation and the phonological loop facilitates the temporary storage (and refreshing) of auditory signals. Later research led to the proposal of an episodic buffer linked to the central executive which, it was suggested, would allow for the 'binding together' of information from the visuospatial sketchpad, phonological loop and long-term memory into a 'unitary episodic representation' (Baddeley 2000; Baddeley 2003: 203). The episodic buffer has a role in long-term episodic learning through the supply and retrieval of information from the episodic long-term memory (Baddeley 2000: 421).

\section{Enactment and memory}

In addition to the possibility that levels of processing might affect memorisation, cognitive psychology research has identified and explored the 'enactment paradigm' which, by obtaining different performance levels to those achieved through verbal-only memorisation, suggested a role for alternative memorisationrelated principles and laws (Nilsson 2000: 137). Whilst initial experiments typically noted superior memory for enacted action phrases, i.e. instructions presented with subject-performed tasks (SPTs) (Engelkamp and Zimmer 1985), other research has noted that merely observing or imagining enactment with real or imagined objects can positively affect recall (Kormi-Nouri 2000). Nilsson (2000: 144) concluded that enactment benefits do not appear to involve adding a 'specific motoric dimension' to memory traces. Explanations for such effects focus, then, on encoding, processing and accessibility.

Cohen $(1981,1983)$ suggested that memory for action events is non-strategic (evidenced by a lack of properties usually associated with word recall: primacy, age and levels of processing effects), and, as such, acted as an optimal form of encoding. In this way, encoding was not reliant upon 'deliberate strategies for remembering' (Nilsson 2000: 137) and has, therefore, been suggested to be of particular use with both younger and learning-impaired learners (Cohen 1989). On the other hand, Nilsson and Bäckman (1989: 174-5 181) proposed qualitative differences in the memory processes for SPTs (compared with verbal tasks) postulating that SPTs involved both explicit and implicit memory components and both (or either) codes could be accessed depending on test conditions. It is, however, important to note that others (Kormi-Nouri 1995) consider the encoding of action events to increase the degree of self-involvement to such an extent that encoding is 'entirely strategic' and facilitated by semantic integration (Nilsson 2000: 139). In other words, enactment is a consciously applied memorisation technique.

Memory for action events might also affect the nature of a memory trace. Enactment during encoding is believed to automatically involve multi-modal processing thereby yielding richer encoding (Bäckman and Nilsson 1984, 1985). Furthermore, motor encoding is reported to be more durable, more accessible and 
'highly resistant to forgetting' (Knopf and Neidhardt 1989: 785). Richer and more distinctive traces are easier to find at recall, and enactment contributes to 'trace distinctiveness', setting a trace 'marker' which aids recall (Cohen and Otterbein 1992). Engelkamp and Zimmer (1989) postulated that SPTs (over visual imagery and watching) promoted superior recall due to a motor system which complemented the verbal and imaginal system. Enactment sufficiently engaged the motor system which contributed to a 'particularly good memory trace' (1989: 153). It has further been suggested that, in line with the Dual Coding Theory of Paivio $(1971,1986)$ which holds that two modalities (visual/verbal) are better than one, three modalities (visual/verbal/motor) should operate more effectively than two (Cohen 1989). The particular benefits of enactment have also been linked to item-specific (rather than relational) motor encoding evidenced by a positive effect for enactment on recognition (Zimmer and Engelkamp 1989) and distinctive motor encoding where an event is 'registered in a multidimensional representational space' (Nilsson 2000: 138).

Furthermore, enactment might also aid retrieval. In other words, as episodic (or event) memory traces comprise bundles of attributes, generation of just one component of a multi-component trace (i.e. an already encoded enactment component) will facilitate retrieval of the whole trace including verbal content (Cohen and Otterbein 1992). Nevertheless, it is important to note that whilst enactment during encoding improves memory performance, enactment during retrieval is considered to be of limited importance (Kormi-Nouri and Nilsson 2001: 100).

\section{Gesture and language}

Gullberg (2006) recognises two key areas which highlight the relevance of gesture in studies of second language acquisition. Firstly, gesture is seen as part of language itself; that is to say, as a developing system for learners to acquire in both production and comprehension terms. Secondly, it is seen as a tool for language acquisition which assists the learner in a number of ways, including: handling expressive difficulties, cross-linguistic (L1/L2) influence and planning and processing. This study will focus on the last of these; more specifically, treating gestures as a means of enhancing memorisation through deeper processing and richer memory traces.

Whilst several frameworks have been proposed for classifying gesture types, Gullberg (2006) notes two distinctive and universal gesture categories: 'emblems' that are codified and conventional, and 'representational or rhythmic gestures' that are non-standard and spontaneous. McCafferty and Stam (2008: 6) see emblems as 'filling a grammatical slot' in language production, and spontaneous gestures as 'thinking-for-speaking' - the interaction between gesture and the organisation of thought to support linguistic encoding. Five key gesture types have been noted by McNeill (1992: 12-18) and are summarised in Table 1. Whilst it is possible that a gesture may belong to multiple categories (McCafferty and Stam 2008: 9), this study will attempt to classify each gesture type used in line with McNeill's well-known framework. 


\begin{tabular}{|c|c|c|}
\hline Gesture type & Function & Example \\
\hline Iconic & $\begin{array}{l}\text { Represent action or object. } \\
\text { Close formal relationship to } \\
\text { the semantic content of speech. }\end{array}$ & $\begin{array}{l}\text { Cup to mouth } \\
=\text { drink }\end{array}$ \\
\hline Metaphoric & $\begin{array}{l}\text { Pictorial. } \\
\text { Relate to an abstraction }\end{array}$ & $\begin{array}{l}\text { Ball shape } \\
=\text { a whole idea }\end{array}$ \\
\hline Deictic & $\begin{array}{l}\text { Identify objects and events } \\
\text { either concrete or abstract. }\end{array}$ & $\begin{array}{l}\text { Thumb over shoulder } \\
=\text { past }\end{array}$ \\
\hline Cohesive & $\begin{array}{l}\text { Tie together themes temporally } \\
\text { separated in discourse. } \\
\text { Emphasise continuities. }\end{array}$ & $\begin{array}{l}\text { Replication of gesture to } \\
\text { take up a point previously } \\
\text { expressed }\end{array}$ \\
\hline Beats & $\begin{array}{l}\text { a. Meta-level of discourse } \\
\text { b. Meta-linguistic - children } \\
<5\end{array}$ & $\begin{array}{l}\text { Nodding to enumerate lists } \\
\text { Accompanying syllable } \\
\text { structure }\end{array}$ \\
\hline
\end{tabular}

Table 1: Gesture classification system after McNeill (1992: 12-18)

\section{Gesture and memorisation}

In the light of research concerning motoric processing, enactment and levels of processing, it seems possible that gestures could act as a form of elaborate memory traces, enhancing memorisation and, ultimately, language learning. In this way, an 'enhanced plus teacher-led' presentation of language (e.g. supported by both visuals and gestures) coupled with explicit instructions to learners to replicate those gestures would increase the likelihood of the learner manipulating depth of processing. This possibility is supported by empirical evidence relating to both first language acquisition and second language learning.

\section{Gesture and L1 acquisition}

Gestures occurring with speech have been shown to facilitate sentence memory by listeners. Early research explored the mnemonic effect of experimenterperformed gesture with first semester university students (Cohen and Otterbein 1992). Meaningful gesture was deemed to significantly affect memorisation of unconnected L1 sentences in both selective and cued recall conditions, when compared with gestures not related to meaning. These researchers concluded that pantomimic gestures (which convey meaning in the absence of verbal speech) acted as 'more powerful retrieval cues' than non-pantomimic gestures, and that these cues could be both sentence-specific and generic (Cohen and Otterbein 1992: 131). In other words, pantomimic gestures aided the recall of specific unconnected sentences and increased recall of gestured rather than non-gestured sentences. Cohen and Otterbein (1992: 132) surmised that adding motor features to memory traces created a more distinctive trace, and that the mnemonic effect of different gestures (pantomimic and non-pantomimic) may well not involve different mechanisms. They added that the usefulness of motor cues might be context-dependent: for example, non-significant gesture findings for connected sentences may indicate that narrative is a strong enough memorisation strategy in its own right, which makes gesture cues redundant.

A later study attempted to explore more specifically the role of gestural meaning in remembering verbal material by comparing experimenter-performed representational and non-representational gestures (hereafter RG and NRG) and 
their respective efficacy with regard to both recall and recognition (Feyereisen 2006: 188). RG contained visual or dynamic features, whilst NRG were deemed to have prosodic relevance (i.e. beats relating to stress modulation). Results demonstrated that recall was facilitated more by RG (gesture matching meaning) than by NRG, and that RG that were 'incongruent with sentence meaning' did not facilitate recall (2006: 198). These findings allowed Feyereisen (2006: 198) to conclude that 'the meaningfulness of the gesture is an important component of the mnemonic effect'.

The hypothesis that gesture could create richer memory traces was explored through the comparison of iconic (meaningful) gesture and visuals with young children aged between 4 and 5 years learning L1 lexical items (Tellier 2005). Results showed that images, rather than iconic gestures, yielded the greatest effect on L1 lexical item recall. It is, however, important to note that the gesture group observed the gestures and did not perform them. Enriched memory traces supporting first language acquisition were also noted through the use of American Sign Language (ASL) with younger learners (Daniels 1997). It was reported that presentation of gestures in the form of ASL enriched African-American preschool children's receptive L1 vocabulary. Daniels (1997: 29) posited that this was due to an additional sensory channel (sign language) which 'augments the usual oral/aural sensory channels' and 'allows a child to feel language'.

\section{Gesture and L2 learning}

Whilst research specifically linking retention rate in L2 lexical learning to gesture use is limited, there is evidence that gestures increase depth of L2 processing resulting in enhanced durability of learning, and also that gestures act as retrieval cues. Allen (1995) found that presentation of emblematic gestures (typical of French culture) alongside semantically-related French expressions significantly affected memorisation (tested through recall) and slowed attrition (tested through successive post-tests) in adult learners. It was posited that greater retention was linked to increased depth of processing and that durability of the mental representation had been affected by presentation of the material to be remembered. Allen (1995) also confirmed that a gesture was an effective retrieval cue, presumably through its links to mental representation. It is, however, relevant that recall scores involved recall of the L1 translation of each L2 sentence, rather than of the L2 sentence. The findings of this study are therefore limited to receptive language ability (listening and understanding).

Later research involving adult participants (mean age 18.5 years) indicated that semantically relevant gestures were instrumental in increasing recall of L2 vocabulary, and that gesture had a direct effect on measureable brain activity (Kelly, McDevitt and Esch 2009). In other words, it was found that gestures enriched encoding rather than merely directing learner attention. In this study, L2 vocabulary learning through iconic gesture was explored to identify whether gestures would enhance learning (both free recall and recognition) due to semantic content rather than for attentional reasons, and also whether a gesturespeech simultaneous multi-modal presentation would be more effective than repetitive speech or uni-modal input. Results supported the contribution of the semantic content of gestures to learning - the congruent gesture plus speech condition producing the highest gains. Possible neural mechanisms which might support learning and remembering new words in a foreign language were 
explored and it was demonstrated that the speech with congruent gestures produced larger Late Positive Complex (LPC) values (reflecting recollection of information in long-term memory) than the speech-only presentation. This, in turn, implied that speech-plus-gesture could affect recall. It was concluded that co-speech gestures 'captured meaning in an embodied and non-arbitrary way' and 'deepened the imagistic memory trace' which enhanced accurate recollection (Kelly et al. 2009: 330).

Subject-performed gestures were also found to be more beneficial than visuals alone in the memorisation of lexical items by younger learners of English as a foreign language (Tellier 2008). Results showed that passive knowledge (word recognition shown by gesture or picture) was equal between groups but that differences in active knowledge (word production) were significant, the gesture group outperforming the picture-only group. From this, Tellier (2008: 911) surmised that gesture production acted as a stronger modality than pictures alone, arguing that involving the body in the learning process created richer memory traces and facilitated recall. The study reported in this paper aims to expand on Tellier's research by examining the memorisation of larger formulaic chunks of language by three age groups of primary school children (ages 4-5, 5-6 and 6-7 years).

To conclude, it would appear that gesture may have a distinct role in language memorisation. Meaningful gestures improve recall (Cohen and Otterbein 1992; Feyereisen 1996; Kelly, McDevitt and Esch 2009) and slow attrition (Allen 1995). Furthermore, a convincing explanation has been offered for younger learners favouring kinaesthetic presentation of language due to developmental considerations (Baker-Ward, Ornstein and Holden 1984; Daniels 1997; Schneider and Sodian 1991; Tellier 2008).

\section{The research questions}

It was noted earlier that younger children's (aged 5-7 years) language learning in the foreign language classroom can appear limited to nouns, basic greeting and songs. Recent research involving slightly older children (aged 7-8 years) showed limited range in oral sentence production and rare production without scaffolding (Cable et al. 2010: 107-109). This study aimed, therefore, to explore the effectiveness of a teaching technique to aid the memorisation of 'meaningful linguistic constructions' or formulaic utterances - unanalysed chunks of language with communicative function (Tomasello 2003: 99). The study addresses the following research questions:

1. Does the use of gesture increase the number of L2 lexical items in formulaic utterances memorised by children aged between 4 and 7 years?

2. Does the use of gesture in memorising L2 lexical items in formulaic utterances reduce attrition (measured by delayed post-test) in children aged between 4 and 7 years?

\section{Method \\ Sample}


This research was conducted with two mixed-year classes covering Year R, Year 1 and Year 2 within a small rural primary school in England. Details of the participating children are provided in Table 2.

\begin{tabular}{|l|l|l|l|l|l|}
\hline \multicolumn{4}{|l|}{ Participants $(N=40)$} & Total \\
\hline Class & \multicolumn{4}{|l|}{ One (mean age 5.4) } & \multicolumn{2}{l|}{ Two (mean age 6.6) } & (mean age 5.10) \\
\hline \multicolumn{4}{|l|}{20} & 20 & 40 \\
\hline Year & Year R & Year 1 & Year 1 & Year 2 & \\
\hline & $10(50 \%)$ & $10(50 \%)$ & $12(60 \%)$ & $8(40 \%)$ & 40 \\
\hline Male & $5(25 \%)$ & $5(25 \%)$ & $7(35 \%)$ & $5(25 \%)$ & $22(55 \%)$ \\
\hline Female & $5(25 \%)$ & $5(25 \%)$ & $5(25 \%)$ & $3(15 \%)$ & $18(45 \%)$ \\
\hline
\end{tabular}

Table 2: Breakdown of sample by class, school year and gender

The mean ages of Class 1 and Class 2 were 5.4 years and 6.6 years respectively (the mean age of the total group was 5.10 years). All the children spoke English as their L1 and all Year 1 and Year 2 children had received approximately 10 weeks' L2 French tuition during the previous summer term (April-July 2009). The Year $\mathrm{R}$ children had not received any formal modern foreign language (MFL) instruction in school. It was also most likely that school provision was the only access these children had to the target language. In this school, French was delivered once a week, as a 45 minute afternoon session.

\section{Procedure}

With the teacher as researcher and, therefore, no opportunity for a control group, this was an action research study. It was, however, rooted in quantitative, statistical analysis and therefore has attempted to adopt as much of the rigour of experimental research as possible. It is hoped that this type of approach will, to some extent, limit potential criticisms of its value as a piece of research rather than purely 'reflective practice' (Nunan 2005: 236).

\section{The pedagogic intervention}

Pinter (2006: 55-56) asserts that the progression of young language learners from listening to speaking is through the use of 'unanalysed chunks' of language which allow faster communication than, for example, word-by-word sentence building. It was also hoped that this study might shed more light on recent longitudinal evidence of limited and static range in respect of sentence production in Year 3 children (Cable, Driscoll, Mitchell and Holmes 2010: 109).

Two very short stories were designed as a vehicle for presenting the target language formulaic utterances, one with supporting gestures, and the other without. The development of the stories centred around a communicative approach to language teaching which would have face validity, concern for realistic contexts, and be learner-centred (Brumfit 2001: 47-54). The two stories were each accompanied by a PowerPoint presentation, in which each slide illustrated a formulaic chunk to be memorised by the participants (see Appendix 1). Whilst the pedagogic intervention aimed to offer participants meaningful communication opportunities, the focus of the study was to evaluate memorisation of target utterances. Therefore, the utterances had to be carefully 
designed, so that the two stories could be closely matched in terms of word count, syllable count and syntactic complexity. Bearing in mind the oral-only presentation of the target language, hyphenated and truncated words (e.g. m'appelle and est-ce) were counted as single words. Story details including word and syllable counts and translations are shown in Table 3.

\begin{tabular}{|l|l|c|c|l|l|l|}
\hline \multicolumn{2}{|l|}{ Slide } & Story 1 (without gestures) & \multicolumn{3}{l|}{ Story Two (with gestures) } \\
\hline 1 & $\begin{array}{l}\text { Bonjour! } \\
\text { Je m'appelle } \\
\text { Georges. } \\
\text { Hello! My name is } \\
\text { George. }\end{array}$ & 4 & 6 & $\begin{array}{l}\text { J'aime jouer à la } \\
\text { marelle. } \\
\text { Ilike playing } \\
\text { hopscotch. }\end{array}$ & 5 & 7 \\
\hline 2 & $\begin{array}{l}\text { Est-ce que tu veux } \\
\text { jouer? } \\
\text { Do you want to } \\
\text { play? }\end{array}$ & 5 & 6 & $\begin{array}{l}\text { Je suis triste. } \\
\text { I'm sad. }\end{array}$ & 3 & 3 \\
\hline 3 & $\begin{array}{l}\text { J'ai marqué } \\
\text { I've scored (a } \\
\text { goal)! }\end{array}$ & 2 & 3 & $\begin{array}{l}\text { Est-ce que tu veux } \\
\text { un tour ? } \\
\text { Do you want a turn ? }\end{array}$ & 6 & 6 \\
\hline 4 & $\begin{array}{l}\text { Nous avons gagné! } \\
\text { We've won! }\end{array}$ & 3 & 5 & $\begin{array}{l}\text { Nous nous } \\
\text { amusons ! } \\
\text { We're having fun ! }\end{array}$ & 3 & 5 \\
\hline
\end{tabular}

Table 3: Comparison of word and syllable counts in language structures

A question with est-ce que was included in each story, previous teaching experience having highlighted younger children's difficulty in learning target language question forms. The text accompanying slide 1 varied slightly in length between stories. This decision, driven mostly by access to visuals, was justified on the grounds that the differences were not substantial and that the shorter, potentially easier utterance (Story 1 , Slide 1 ) would be incorporated into the story without gesture.

Appendix 1 shows the visuals and language structures for each story. At the beginning of each presentation, the communicative intention of each slide was elicited from the students (in their L1) to ensure that the children understood the meaning of the utterances they were attempting to memorise (Gower and Walters 1983: 110). When the stories were subsequently told to the children in French, Story 1 was accompanied by visuals only, while each utterance in Story 2 was supported by visuals plus gestures which aimed to give some kind of elaboration (Appendix 2). Various gesture types were included and whilst most were iconic and therefore supported semantic elaboration, a gesture was occasionally used which aimed to form an association between similar L1 and L2 phonological representations. Each word was gestured at syllable level to account for learners relying on oral presentation and therefore the possibility that they would recognise syllables rather than whole words in the speech stream. Table 4 attempts to classify each gesture within McNeil's classification system (McNeil 1992: 12-18), with the exception of the keyword gesture. 


\begin{tabular}{|c|c|c|c|}
\hline Slide No: & Word: & Gesture: & Gesture type: \\
\hline \multirow[t]{5}{*}{1} & J'aime & Thumbs up & Iconic \\
\hline & jouer & Moving hands out \& in & Iconic \\
\hline & $\bar{a}$ & Make letter 'A' shape & Metaphoric \\
\hline & $l a$ & Make letter 'L' shape & Metaphoric \\
\hline & marelle & $\begin{array}{l}\text { One finger then two } \\
\text { imitating hopscotch }\end{array}$ & Iconic \\
\hline \multirow[t]{3}{*}{2} & Je & Point to self & Deictic \\
\hline & suis & Sweeping movement & Keyword \\
\hline & triste & Mime crying & Iconic \\
\hline \multirow[t]{5}{*}{3} & Est-ce que & $\begin{array}{l}\text { Hands raised palms } \\
\text { upwards - 'why?' x } 2\end{array}$ & Iconic \\
\hline & $T u$ & Point to other & Deictic \\
\hline & veux & Make letter 'V' shape & Metaphoric \\
\hline & un & Hand 'under' other hand & Keyword \\
\hline & tour & Circle with finger 'tour' & Keyword \\
\hline \multirow[t]{3}{*}{4} & Nous & Make letter ' $N$ ' shape & Metaphoric \\
\hline & nous & Make letter ' $N$ ' shape & Metaphoric \\
\hline & amusons & Punching air x 3 & Iconic \\
\hline
\end{tabular}

Table 4: Table showing types of gesture

\section{Reliability and validity}

This study involved convenience sampling as two intact classes took part, each receiving three weeks' instruction using the pedagogical intervention outlined above. In a brief and informal pre-test of the children's existing L2 knowledge, some children $(n=8)$ correctly identified either bonjour! or je m'appelle, or both. It was decided that, as this language formed part of the non-gestured story, no serious bias would result.

Both stories were presented in the same way to both classes, during three consecutive lessons (once a week). The second and third teaching session and the post-test and delayed post-test were video-recorded for analysis and a strict protocol was followed for each presentation. An identical sequence was followed for both stories; each story was told using the exact scripted words, accompanied by the visuals. In Story 2 each presentation was also accompanied by teacher gestures which the children were explicitly encouraged to replicate.

Group oral post-tests (four participants per group) were conducted immediately following the third session and group oral delayed post-tests 
performed two weeks after the third session. Both required cued recall of the correct L2 formulaic utterances. That is, in both test conditions, the groups were required to view each slide once then attempt to produce the correct formulaic chunk. (Unfortunately there was a high rate of absenteeism on the day of the third teaching session and post-test, which reduced to 24 the number of children in the final sample discussed in the results section below).

The children were tested in identical groups at post-test and delayed post-test. Whilst it was possible that group testing might lead to stronger pupils supporting weaker ones, it was felt that such a procedure would minimise nerves and fear of making mistakes and encourage a supportive and collaborative experience where the optimum amount of language might be elicited. Pinter (2006: 147) advises similar procedures for interviewing young learners in order to avoid possible overwhelming effects of one-on-one interaction. As both tests were videorecorded, it would still be possible to separate out each child's performance.

\section{Data analysis}

The test video evidence was viewed by the teacher-researcher retrospectively and memorisation of target language was quantitatively analysed. It was decided to count the number of complete individual words accurately produced by each child, as the best indicator of lexical recall. Video data was cross marked by a teaching assistant and inter-rater reliability was calculated at $90.3 \%$.

\section{Results}

\section{Post-test results - Story 1 and Story 2}

The table below (Table 5) shows individual test scores at post-test of the 24 children participating in all aspects of the study, for both stories (number of words recalled per story).

\begin{tabular}{|c|c|c|c|}
\hline Participant no & $\begin{array}{c}\text { Words recalled } \\
\text { Story 1 } \\
\text { (no gestures) }\end{array}$ & $\begin{array}{c}\text { Words recalled } \\
\text { Story 2 } \\
\text { (gesture) }\end{array}$ & $\begin{array}{c}\text { Difference } \\
\text { S1/S2 }\end{array}$ \\
\hline 3 & 4 & 14 & +10 \\
\hline 6 & 0 & 14 & +14 \\
\hline 7 & 9 & 16 & +7 \\
\hline 8 & 11 & 16 & +5 \\
\hline 9 & 4 & 0 & -4 \\
\hline 11 & 4 & 10 & +6 \\
\hline 12 & 4 & 9 & +5 \\
\hline 13 & 6 & 9 & +3 \\
\hline 17 & 4 & 4 & 0 \\
\hline 18 & 9 & 16 & +7 \\
\hline 19 & 0 & 0 & 0 \\
\hline 21 & 7 & 10 & +3 \\
\hline 22 & 2 & 3 & +1 \\
\hline 24 & 4 & 6 & +2 \\
\hline 25 & 0 & 14 & +14 \\
\hline 27 & 6 & 16 & +10 \\
\hline 29 & 11 & 15 & +4 \\
\hline 30 & 0 & 0 & 0 \\
\hline
\end{tabular}




\begin{tabular}{|c|c|c|c|}
\hline 32 & 6 & 15 & +9 \\
\hline 33 & 6 & 3 & -3 \\
\hline 34 & 3 & 3 & 0 \\
\hline 35 & 0 & 12 & +12 \\
\hline 37 & 2 & 5 & +3 \\
\hline 40 & 6 & 10 & +4 \\
\hline
\end{tabular}

Table 5: Participant scores at post-test

Figure 1 illustrates the distribution of scores achieved across the sample for Story 1 (no gestures). A perfect score for this story would have been recall of 14 lexical items. Results show a mean score of 5.68 items ( $s d=2.709$, range $=9)$. The lowest score of 2 represented $14.3 \%$ of the total possible and the highest score of 11 , $78.6 \%$ of the total.

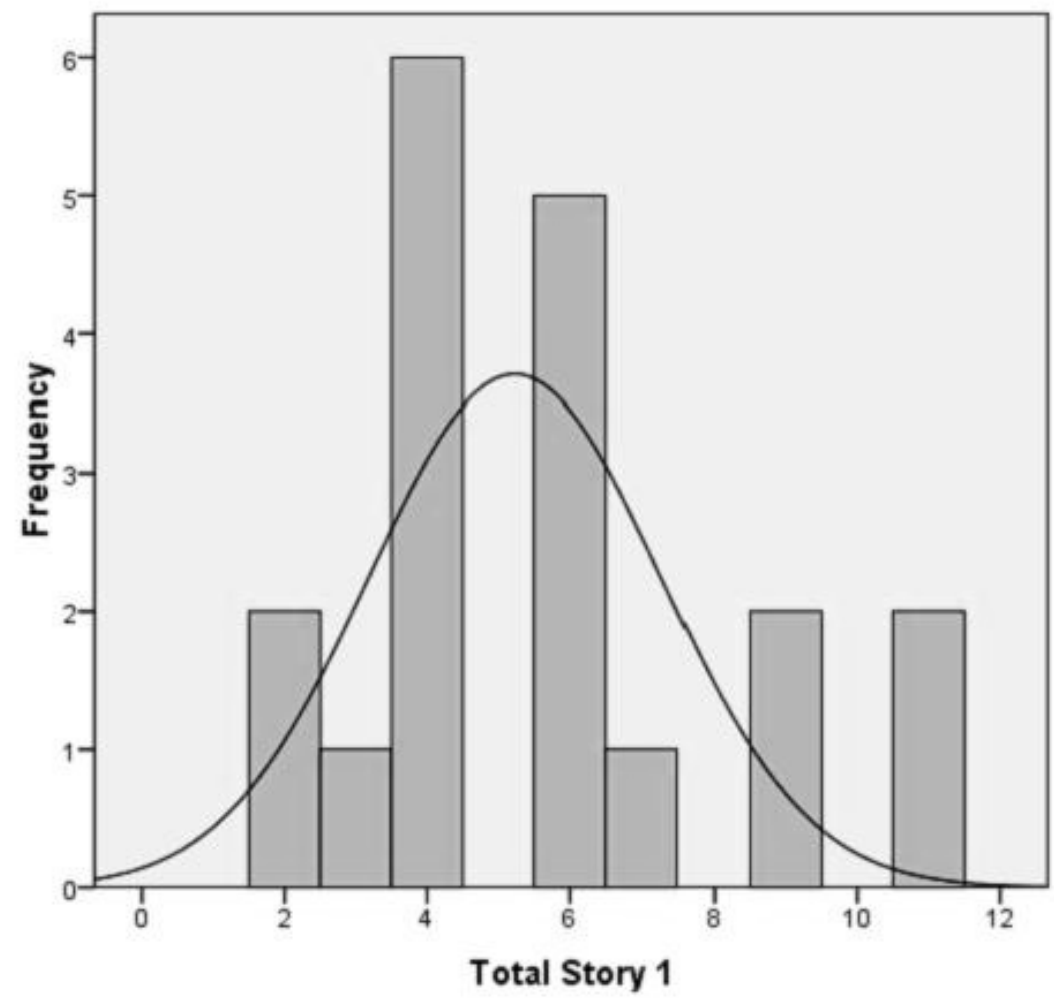
$\begin{aligned} \text { Mean } & =5.68 \\ \text { Std. Dev. } & =2.709\end{aligned}$ $\mathrm{N}=19$

Figure 1: Histogram showing samples scores for Story 1 at post-test

Figure 2 shows the distribution of scores achieved across the sample for Story 2 (with gestures). A perfect score for this story would have been recall of 17 lexical items. Results show a mean score of 10.48 items ( $s d=4.833$, range $=13$, $\min =3$ or $17.6 \%$ of the total, $\max =16$ or $94.1 \%$ of the total); however the histogram indicates a modal score of 16 . The distribution was negatively skewed (skewness value -.387) and therefore tended towards the higher range. 


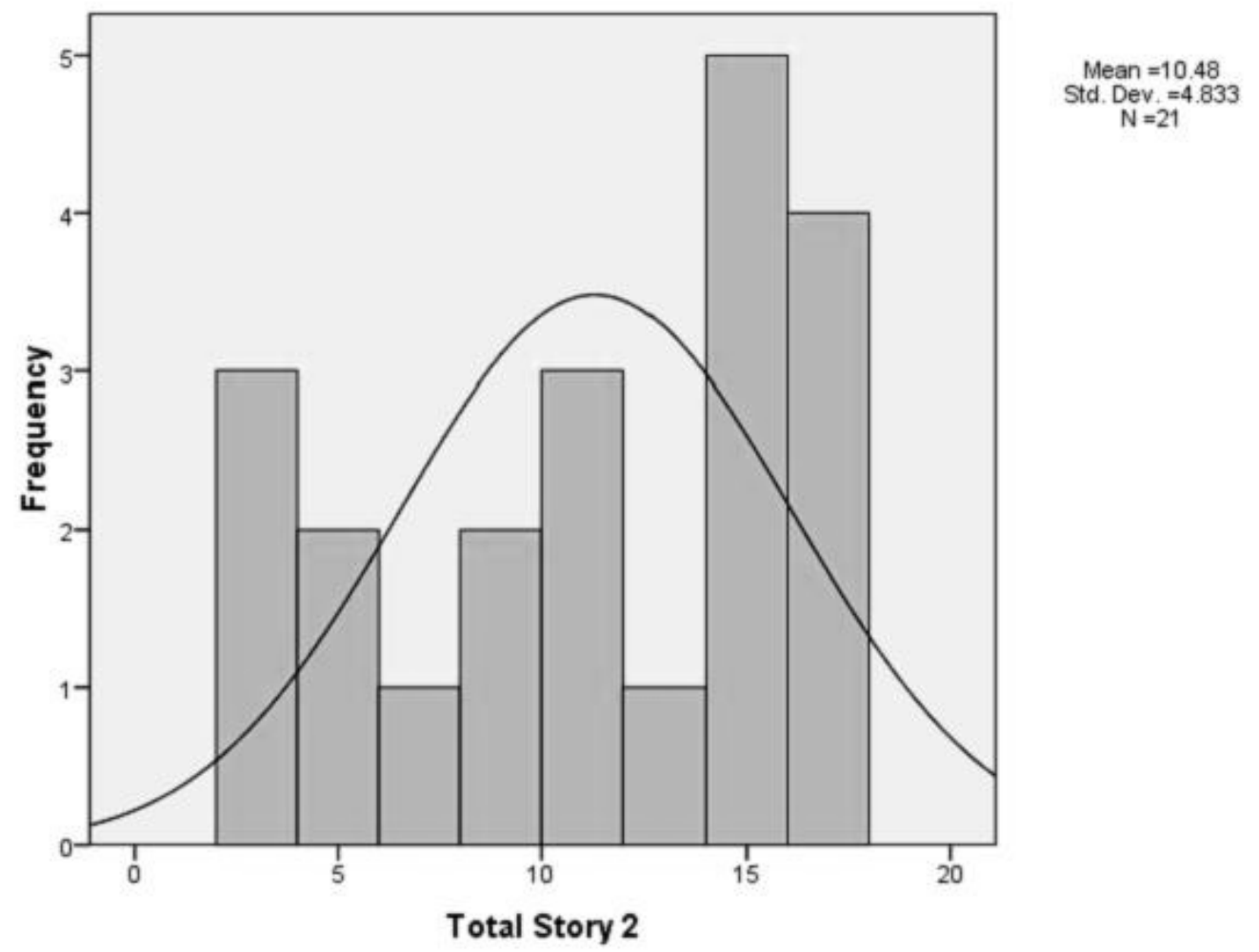

Figure 2: Histogram showing sample scores for Story 2 at post-test

A paired samples $t$-test (from standardised scores) revealed significant difference in lexical recall between the non-gestured and gestured stories at post-test $(t=-$ 3.385, $d f=17$, asymp. sig. $=.004)$. A small effect size (0.33) was calculated using $\eta^{2}$ statistic. In other words, the participants showed significantly better memorisation of the story told with gestures.

\section{Delayed post-test results - Story 1 and Story 2}

Table 6 shows individual raw scores at delayed post-test for both stories, again for the 24 children participating in all aspects of the study.

\begin{tabular}{|l|l|l|l|}
\hline Participant no & $\begin{array}{c}\text { Words recalled } \\
\text { Story 1 } \\
\text { (no gestures) }\end{array}$ & $\begin{array}{c}\text { Words recalled } \\
\text { Story 2 } \\
\text { (gestures) }\end{array}$ & $\begin{array}{c}\text { Difference } \\
\text { S1/S2 }\end{array}$ \\
\hline 3 & 4 & 7 & +3 \\
\hline 6 & 4 & 4 & 0 \\
\hline 7 & 6 & 12 & +6 \\
\hline 8 & 4 & 11 & +7 \\
\hline 9 & 4 & 11 & +7 \\
\hline
\end{tabular}




\begin{tabular}{|l|l|l|l|}
\hline 11 & 6 & 11 & +5 \\
\hline 12 & 7 & 11 & +4 \\
\hline 13 & 4 & 11 & +7 \\
\hline 17 & 4 & 2 & -2 \\
\hline 18 & 7 & 11 & +4 \\
\hline 19 & 0 & 0 & 0 \\
\hline 21 & 4 & 6 & +2 \\
\hline 22 & 4 & 2 & -2 \\
\hline 24 & 4 & 11 & +7 \\
\hline 25 & 7 & 3 & -4 \\
\hline 27 & 6 & 12 & +6 \\
\hline 29 & 4 & 3 & -1 \\
\hline 30 & 4 & 9 & +5 \\
\hline 32 & 0 & 0 & 0 \\
\hline 33 & 6 & 4 & -2 \\
\hline 34 & 0 & 0 & 0 \\
\hline 35 & 9 & 3 & -6 \\
\hline 37 & 0 & 8 & +8 \\
\hline 40 & 0 & 0 & 0 \\
\hline
\end{tabular}

Table 6: Comparison of Story 1 and Story 2 raw scores at delayed post-test

Figure 3 shows the distribution of scores achieved across the sample for Story 1 (no gestures) at delayed post-test. A perfect score for this story would have been recall of 14 lexical items. Results show a mean score of 5.16 items ( $s d=1.537$, range $=5, \min =4$ or $28.6 \%, \max =9$ or $64.3 \%$ ). The distribution was positively skewed (skewness value 1.039) towards the lower range. 


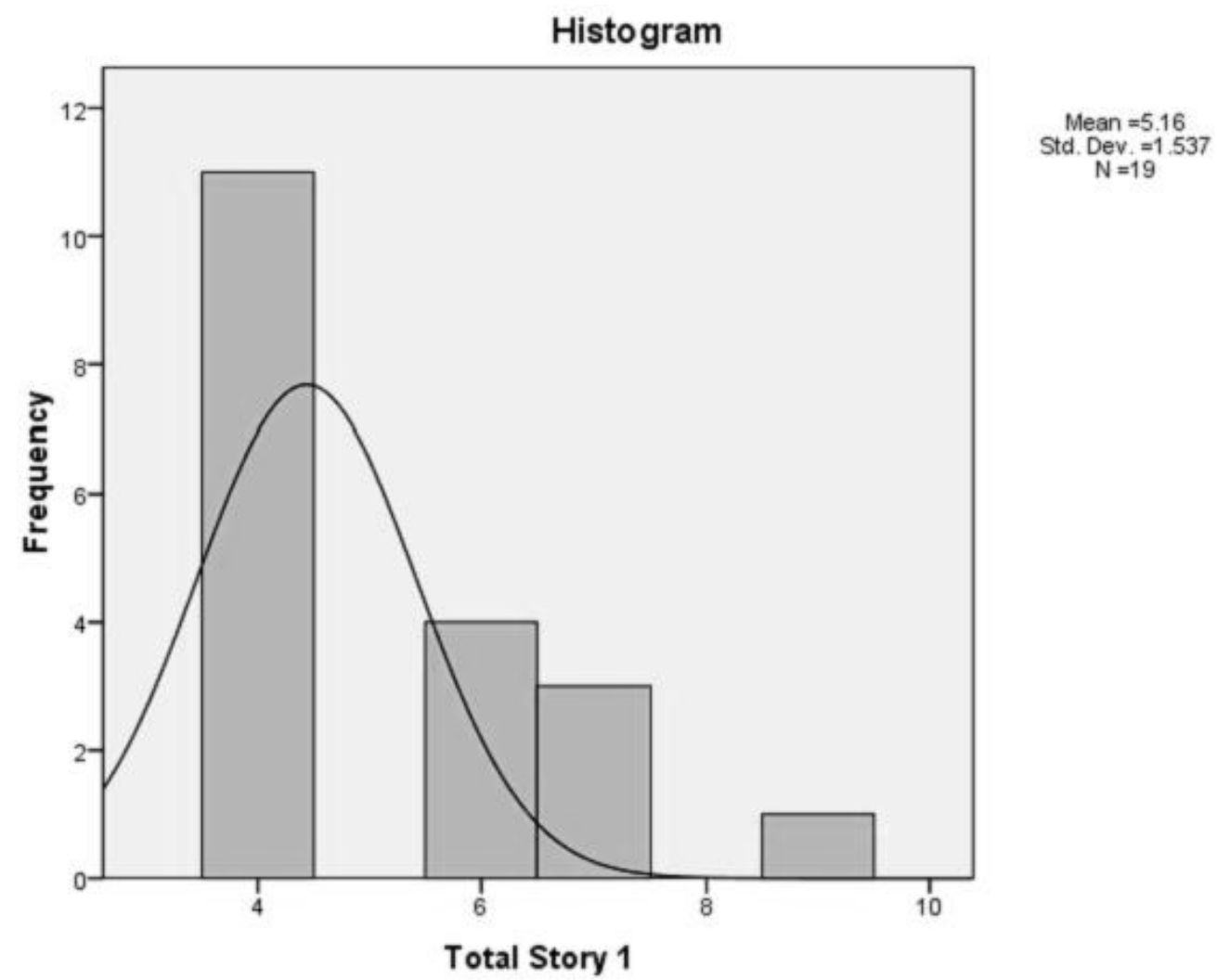

Figure 3: Histogram showing scores for Story 1 at delayed post-test

Figure 4 (below) shows the distribution of scores achieved across the sample for Story 2. A perfect score for this story would have been recall of 17 lexical items. Results show a mean score of 7.60 items ( $s d=3.817$, range $=10, \min =2$ or $11.8 \%$, $\max =12$ or $70.6 \%$ ). The distribution was negatively skewed (skewness value .308) towards the upper range. 


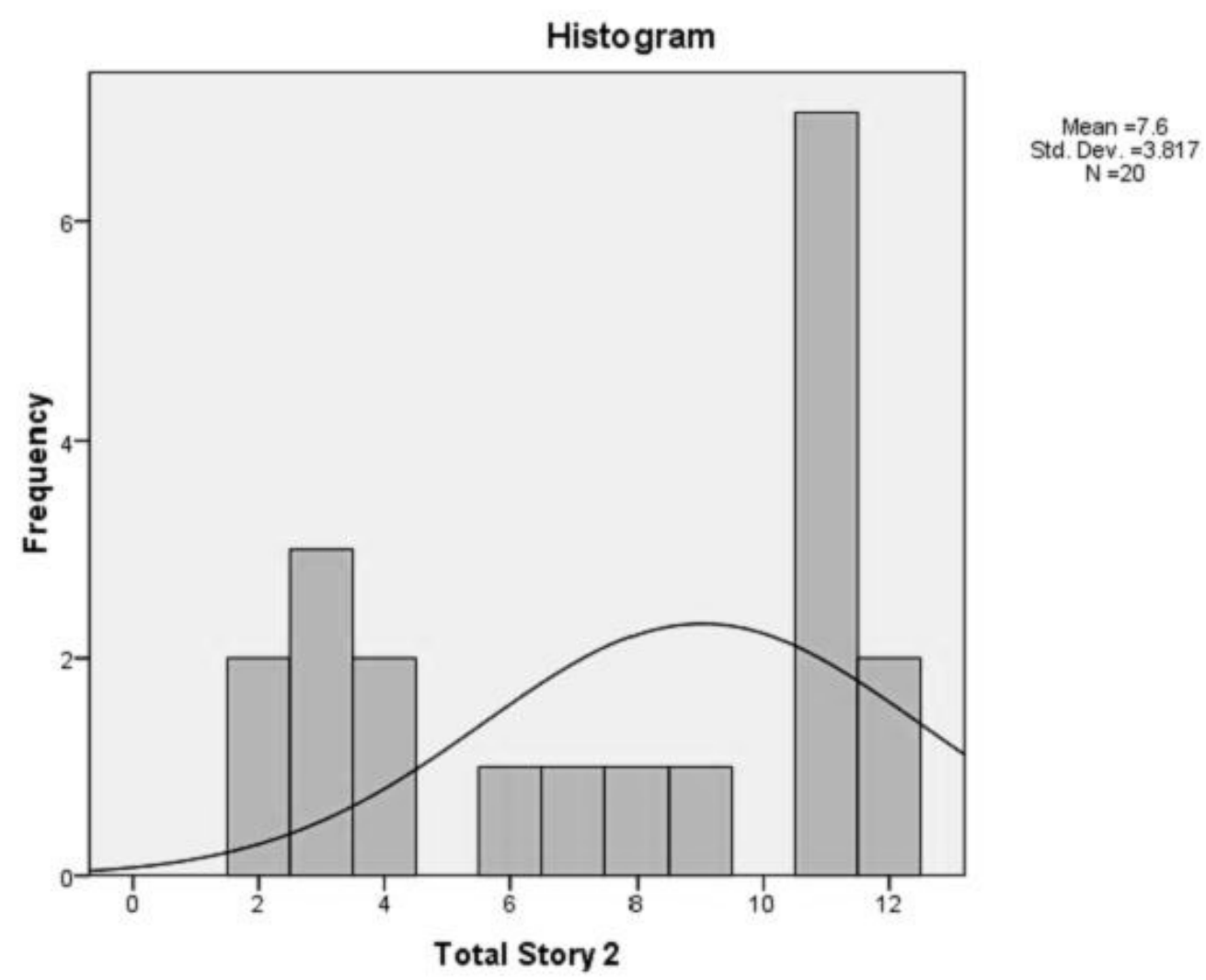

Figure 4: Histogram showing sample scores for Story 2 at delayed post-test

A paired samples $t$-test (from standardised scores) did not reveal significant difference between the non-gestured and gestured stories at delayed post-test $(t=-1.327, d f=18$, asymp. sig. $=.201)$. In other words, memorisation was no longer greater for the gestured story two weeks after the presentation.

\section{Discussion}

\section{Research question 1}

Does the use of gesture increase the number of L2 lexical items in formulaic utterances memorised by children aged between 4 and 7 years?

The large difference in mean scores at post-test between the non-gestured Story 1 (mean 5.68) and the gestured Story 2 (mean 10.48) indicates that, in this study, learning formulaic utterances combined with gestures aided memorisation. The best participants were able to recall $94.1 \%$ of Story 2 (gestured) compared with $78.6 \%$ of Story 1 (non-gestured). The overall impression then is that the young learners who participated in this research clearly responded favourably (in terms of memorisation) to the use of gestures.

These findings would appear to support opinion that elaboration of material at the time of presentation achieves greater depth of processing measured by 
greater retention (Allen 1995) and that participant-performed gesture supports word production and shows gains in long-term memory (Tellier 2008). However, it is important to note that, whilst mean scores generally improved, performance across the sample was variable. Nevertheless, comparison involving slide matching appeared to support evidence of more accessible memory traces across the sample in the gestured condition; 17 had partial or full recall of slide three in Story 2, compared to 4 participants for the comparable slide in Story 1. This seems to support the possibility that a more reliable memory trace is formed reliability of access being deemed to distinguish recall from recognition (Baddeley 1997: 193). This idea of reliability of memory trace echoes Allen's (1995: 527) assertion that greater recall was a 'measurable indication of depth of processing and of more durable mental representations' [my italics].

\section{Research question 2}

Does the use of gesture in memorising L2 lexical items affect attrition (measured by delayed post-test) within children aged between 4 and 7 years?

The difference in mean scores even at delayed post-test between the non-gestured Story 1 (mean 5.16) and the gestured Story 2 (mean 7.60) might indicate that attrition was slowed by formulaic utterances combined with gestures. The best participants were able to recall $70.6 \%$ of Story 2 (gestured) compared with $64.3 \%$ of Story 1(non-gestured). However, in contrast to the immediate post-test, this difference was not statistically significant for the group as a whole. In other words, the sample responded favourably (in terms of memorisation) to gesture but the gap between gestured and non-gestured performance had narrowed by delayed post-test.

These findings would appear to support the view that elaboration of material at the time of presentation achieves greater depth of processing (if greater retention is an accurate indicator of increased processing depth) and furthermore that the manner of original presentation of linguistic material might affect the development and durability of mental representations (Allen 1995). However, whilst participants retained more of Story 2 at delayed post-test, within-story attrition appeared to considerably favour the non-gestured condition. The rate of attrition for Story $1(0.52)$ equated to a $12.3 \%$ reduction; at 2.88 for Story 2, the rate more than doubled to 27.5\%: in other words, the attrition rate for Story 2 was approximately four times that of Story 1 . These findings might imply that the memorisation gains supported gesture at post-test are boosted through elaborated processing, but that without repetition and retrieval practice, this leads to a sharper drop as memorisation recedes to a more usual level.

\section{Enriched and more accessible memory traces}

Memorisation theory holds that recall (the indicator of memorisation used in this study) depends on accessibility rather than storage. Tulving and Osler (1968) and Tulving and Thomson (1973) posit instead that retrieval cues play a key role in memorisation. Effective retrieval cues are determined by specific encoding operations which adapt the memory trace of an event (Tulving and Thomson 1973: 370). The memory trace, they argue, is a link between the encoding conditions and recall environment and both recall and recognition are 'influenced 
greatly by the nature of information present in the retrieval environment' (Tulving and Thomson 1973: 370).

Following Tellier's (2008) study which examined the effects of gestures and visuals as separate variables, it could be argued that, in this study, both stories have elaborated presentation. It follows that Story 2, as a visuals-plus-gesture condition, is a form of 'elaborated-plus' presentation and this might therefore account for the particularly successful post-test scores for this test, in line with claims identified in the introduction that deeper encoding led to higher retention of lexical items (Hulstjin 2001) and that a deepened imagistic memory trace enhances accurate recollection (Kelly et al. 2009: 330). Furthermore, Story 1 scores might also have been boosted as the story one condition is also elaborated through visuals. It seems possible, therefore, that the overall marked difference in performance between the elaborated and the elaborated-plus conditions is related to the degree of learner involvement. More specifically, whilst gesture might create deeper encoding, it also acts as a retrieval cue in similar ways to the keyword technique where an L2 word is linked acoustically to an L1 word or syllable which is then represented by gesture:

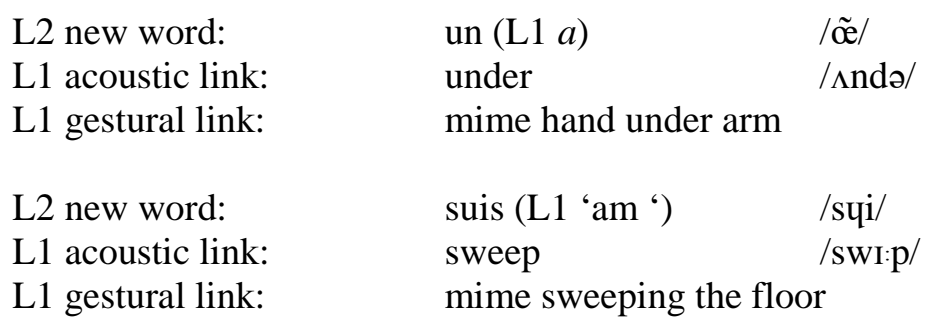

Enacting demands involvement and directs attention; both are especially important with young learners. It is suggested that, in this instance, involvement might create experience or 'conscious awareness' (Baddeley 1997: 205). Retrieval cues are important in memorisation because they 'can be used to evoke specific memories' and 'manipulate the probability that an item will or will not be accessible to the subject' (Baddeley 1997: 194, 210). Paivio (2006) proposes a Dual Coding Theory Conceptual Peg Hypothesis which supports the idea of a 'concreteness advantage' in language memory - that concrete memory exceeds abstract memory performance by a 2:1 ratio. Furthermore, as we have seen, Paivio proposes that compound images (in this study gesture/word) form pairs during presentation, from which a concrete stimulus (gesture) cues recall of its pair (word).

Clearly, though, not all of the gestures in this study were keyword-type gestures based on a degree of phonological similarity with existing L1 representations. It could be possible, then, that whilst gestures may benefit memorisation, their effect is complex and multi-faceted; they may be capable of acting as retrieval cue and semantic elaboration (e.g. je - pointing to self) but not always as both. This is supported by Hulstjin's view that encoding relating to pronunciation can be just as successful as that relating to semantics (Hulstjin 2001: 270). Contrasting different effects from various types of gesture has not been a primary aim of this study and, therefore, those variables have not been rigorously controlled for. It could, however, form the basis of future research. 


\section{The importance of repetition}

Whilst considering the benefits that forms of elaborated processing might bring to the acquisition of L2 lexical items, it is important to also consider the role of repetition. The 'with-gesture subjects' in Tellier's study (2008) scored means of 3.1, 3.7 and 3.8 out of a maximum of eight lexical items across three consecutive test sessions. This study's mean at post-test for Story 2 (gestured) was 10.48 at post-test out of a possible 17 items. It is posited that underlying both the test-ontest increases (Tellier 2008) and the superior gains of this study, is the opportunity for practice and repetition which, alongside deeper processing, forms an integral part of the learning process.

Both Nilsson, Cohen and Nyberg (1989) and Knopf and Neidhardt (1989) note that SPT effects only last in the short term, after which enhanced SPT or verbal learning attrition progresses at the same rate. Ellis (1995) considers that for effective productive vocabulary learning, the keyword technique must be complemented with repetition practice. He argues that successful retrieval strengthens the recalled item and, therefore, can facilitate long term memory as much or more than another learning presentation (expanded rehearsal). Hulstjin (2001: 269) argues that rich, elaborate processing must be reactivated through rehearsal activity (either repetition or retrieval practice) in order to create lasting traces. Both this study and that of Tellier (2008) seem to support these claims.

\section{Conclusion}

This study has built on existing research into gesture and memorisation in L2 learners by attempting to explore the memorisation of words introduced through formulaic chunks (rather than nouns in isolation as previously explored by Tellier 2008). The study exposed the same group of learners to two different instructional conditions, and the learning outcomes of the two conditions were compared. Future research would ideally incorporate a quasi-experimental approach with separate control and experimental groups in order to produce more reliable and generalisable findings. Consideration could also be given to the impact of gesture type and to the importance of subject-performed gesture within the sample.

The results from this study show clear advantage for the memorisation of formulaic chunks of language with gesture-elaborated encoding in very young learners. This was demonstrated by a post-test mean score for the gestured story which was almost double that of its non-gestured counterpart. Further, bearing in mind that both presentations were elaborated (one with visuals; the other with visuals plus gesture), an argument could be made for the advantages of involving young children in second language learning through enacting, rather than purely observing, elaborated presentations. Nevertheless, elaborated traces need to be supplemented with regular repetition of the target language and opportunities for retrieval practice. This was supported by data which showed an attrition rate for the gestured story which was almost twice that of the non-gestured story. In other words, second language vocabulary should constantly be revisited, renewed and refreshed in order to result in longer-term learning. Enhanced elaboration alone is not enough to compete with the effects of attrition. 


\section{Acknowledgements}

I would like to thank Professor Ros Mitchell and Dr Sarah Rule for their advice and comments on earlier drafts of this paper. Story images are reproduced by kind permission of Phillip Martin.

\section{References}

Allen, L. 1995. The effects of emblematic gestures on the development and access of mental representations of French expressions. The Modern Language Journal, 79: 521-529.

Asset Languages and CILT 2010. Making and Marking Progress on the DCSF Languages Ladder.

Atkinson, R.C. and M.R. Raugh 1975. An application of the mnemonic keyword method to the acquisition of a Russian vocabulary. Journal of Experimental Psychology: Human Learning and Memory 104, no.2: 126-133.

Bäckman, L. and L-G. Nilsson 1984. Aging effects in free recall: an exception to the rule. Human Learning, 3: 53-69.

Bäckman, L. and L-G. Nilsson 1985. Prerequisites for lack of age differences in memory performance. Experimental Aging Research 11: 67-73.

Baddeley, A.D. 2000. The episodic buffer: a new component of working memory? Trends in Cognitive Sciences, 4: 417-423.

Baddeley, A. 1997. Human Memory: Theory and Practice. Revised edition. Hove, UK: Psychology Press.

Baddeley, A.D. and Hitch, G.J. 1974. Working memory. In Recent Advances in Learning and Motivation ed. G.A. Bower, vol. 8: 47-90. New York: Academic Press.

Baker-Ward, L., P.A. Ornstein and D. J. Holden. 1984. The expression of memorisation in early childhood. Journal of Experimental Child Psychology, 37, 3: 555-575.

Brumfit, C. 2001. Individual Freedom in Language Teaching. Oxford: Oxford University Press.

Department for Children, Schools and Families 2010. Key Stage 2: A Longitudinal Study. Final Report (DCSF Research Report - RR198). London: DCSF.

Canale, M. and M. Swain. 1980. Theoretical bases of communicative approaches to second language teaching and testing. Applied Linguistics 1: 1-47.

Cohen, R.L. 1981. On the generality of some memory laws. Scandinavian Journal of Psychology 22: 267-281.

Cohen, R.L. 1983. The effect of encoding variables on the free recall of words and action events. Memory and Cognition 11, no.6: 575-582.

Cohen, R.L. 1989. Memory for action events: the power of enactment. Educational Psychology Review 1: 57-80.

Cohen, G., G. Kiss and M. Le Voi. 1993. Memory: Current Issues. Buckingham, UK: Open University Press.

Cohen, L., L. Manion and K. Morrison. 2007. Research Methods in Education. Abingdon, UK: Routledge.

Cohen, R. and N. Otterbein. 1992. The mnemonic effect of speech gestures: pantomimic and non-pantomimic gestures compared. European Journal of Cognitive Psychology, 4, 2: 113-139.

Craik, F.I.M. and R.S. Lockhart. 1972. Levels of processing: a framework for memory research. Journal of Verbal Learning and Verbal Behavior 11: 671-684.

Daniels, M. 1997. Teacher enrichment of pre-kindergarten curriculum with sign language. Journal of Research in Childhood Education 12: 27-33.

Department for Education and Skills (DfES) 2005. The Key Stage 2 Framework for Languages. Nottingham, UK: DfES Publications. 
Ellis, N.C. 1995. The psychology of foreign language vocabulary acquisition: implications for CALL. Computer Assisted Language Learning Journal, 8: 103-128.

Ellis, N.C. 2001. Memory for language. In Cognition and Second Language Instruction ed. P. Robinson, 33-68. Cambridge: Cambridge University Press.

Engelkamp, J. and H.D. Zimmer. 1985. Motor programs and their relation to semantic memory. German Journal of Psychology, 9: 239-254.

Engelkamp, J. and H.D. Zimmer. 1989. Memory for action events: a new field of research. Psychological Research, 51: 153-157.

Feyereisen, P. 2006. Further investigation on the mnemonic effect of gestures: their meaning matters. European Journal of Cognitive Psychology 18: 185-205.

Gower, R. and S. Walters. 1983. Teaching Practice Handbook: a Reference Book for EFL Teachers in Training. Oxford: Heinemann International.

Gullberg, M. 2006. Some reasons for studying gesture and second language acquisition (Hommage à Adam Kendon). International Review of Applied Linguistics in Language Teaching, 44: 103-124.

Hulstjin, J.H. 2001. Intentional and incidental second language vocabulary learning: a reappraisal of elaboration, rehearsal and automaticity. In Cognition and Second Language Instruction ed. P. Robinson, 258-286. Cambridge: Cambridge University Press.

Kelly, S.D., T. McDevitt and M. Esch. 2009. Brief training with co-speech gesture lends a hand to word learning in a foreign language. Language and Cognitive Processes 204: 313-334.

Kormi-Nouri, R. 1995. The nature of memory for action events: an episodic integration view. European Journal of Cognitive Psychology, 7: 337-363.

Kormi-Nouri, R. 2000. The role of movement and object in action memory: a comparative study between blind, blindfolded and sighted subjects. Scandinavian Journal of Psychology, 41: 1: 71-5.

Kormi-Nouri, R. and L-G. Nilsson. 2001. The motor component is not crucial! In Memory for Action: a Distinct Form of Episodic Memory? ed. H.D. Zimmer, R.L. Cohen, M.J. Guynn, J. Engelkamp, R. Kormi-Nouri and M-A Foley, 136-143. Oxford: Oxford University Press.

Knopf, M. and E. Neidhardt. 1989. Aging and memory for action events: the role of familiarity. Developmental Psychology 25: 780-786.

Mackey, A. and S.M. Gass. 2005. Second Language Research Methodology and Design. Mahwah, NJ: Lawrence Erlbaum.

McCafferty, S.G. 2004. Space for cognition: gesture and second language learning. International Journal of Applied Linguistics 14: 1.

McCafferty, S.G. and G. Stam. 2008. Gesture studies and second language acquisition: a review. In Gesture: Second Language Acquisition and Classroom Research, ed. S.G. McCafferty and G. Stam, 3-24. Abingdon, UK: Routledge.

Moore, J.C. and J.R. Surber. 1992. Effects of context and keyword methods on second language vocabulary acquisition. Contemporary Educational Psychology 17: 286292.

Nilsson, L-G. 2000. Remembering actions and words. In The Oxford Handbook of Memory, ed. E. Tulving and F.I.M. Craik, 137-148. New York: Oxford University Press.

Nilsson, L-G. and L. Bäckman. 1989. Implicit memory and the enactment of verbal instructions. In Implicit Memory: Theoretical Issues, ed. S. Lewandowsky, J. Dunn and K. Kirsner. 1989. Hillsdale, NJ: Erlbaum.

Nilsson, L-G., R.L. Cohen and L. Nyberg. 1989. Recall of enacted and nonenacted instructions compared: forgetting functions. Psychological Research, 51: 188-193.

Nunan, D. 2005. Classroom research. In Handbook of Research in Second Language Teaching and Learning ed. E. Hinkel, 225-240. Mahwah, NJ: Lawrence Erlbaum.

Paivio, A. 1971. Imagery and Verbal Processes. New York: Holt, Rinehart and Winston. 
Paivio, A. 1986. Mental Representations: A Dual Coding Approach. Oxford: Oxford University Press.

Paivio, A. 2006. Mind and its Evolution: A Dual Coding Theoretical Interpretation. Mahwah, NJ: Lawrence Erlbaum.

Pinter, A. 2006. Teaching Young Language Learners. Oxford: Oxford University Press.

Robinson, P. (ed.) 2002. Individual Differences and Instructed Language Learning. Amsterdam: John Benjamins.

Schneider, W. and B. Sodian. 1991. A longitudinal study of young children's memory behaviour and performance in a sort-recall task. Journal of Experimental Child Psychology, 51: 14-29.

Tellier, M. 2005. L'utilisation des gestes en classe de langue: comment évaluer leur effet sur la mémorisation du lexique? In Actes du Colloque International de Didactique Cognitive, DidCog 2005. ed. M. Billières, P. Gaillard, and N Spanghero-Gaillard, 26/28 janvier 2005. CD-Rom. Toulouse.Université de Toulouse-Le Mirail.

Tellier, M. 2006. L'impact du geste pédagogique sur l'enseignement/apprentissage des langues étrangères. Thèse de doctorat. Université Paris 7 - Denis Diderot, UFR Linguistique.

Tellier, M. 2008. The effect of gestures on second language memorisation by young children. Gesture, 8: 219-235.

Tomasello, T. 2003. Constructing a Language: A Usage-Based Theory of Language Acquisition. Cambridge, MA: Harvard University Press.

Tulving, E. and S. Osler. 1968. Effectiveness of retrieval cues in memory for words. Journal of Experimental Psychology, 77, no.4: 593-601.

Tulving, E. and D.M. Thomson. 1973. Encoding specificity and retrieval processes in episodic memory. Psychological Review, 80: 352-373.

Zimmer, H.D. and J. Engelkamp. 1989. Does motor encoding enhance relational information? Psychological Research, 51: 158-167. 